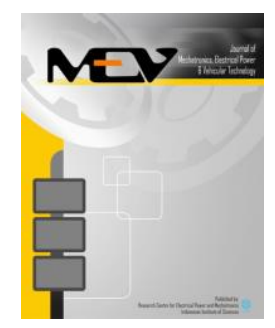

Journal of Mechatronics, Electrical Power, and Vehicular Technology

e-ISSN:2088-6985

p-ISSN: 2087-3379

\title{
Nonlinear Tracking Control of a 3-D OVERHEAd Crane WITH FRICTION AND PAYLOAD COMPENSATIONS
}

\author{
Anh-Huy Vo a , Quoc-Toan Truong a ${ }^{\text {a }}$ Ha-Quang-Thinh Ngo ${ }^{\text {a,b }}$, Quoc-Chi Nguyen ${ }^{\text {a,b,* }}$ \\ ${ }^{a}$ Department of Mechatronics, Ho Chi Minh City University of Technology \\ 268 Ly Thuong Kiet st., Dist. 10, 703500, Ho Chi Minh City, Vietnam \\ ${ }^{\mathrm{b}}$ Control and Automation Laboratory, Ho Chi Minh City University of Technology \\ 268 Ly Thuong Kiet st., Dist. 10, 703500, Ho Chi Minh City, Vietnam
}

Received 29 February 2015; received in revised form 02 May 2015; accepted 02 May 2015

Published online 29 July 2016

\begin{abstract}
In this paper, a nonlinear adaptive control of a 3D overhead crane is investigated. A dynamic model of the overhead crane was developed, where the crane system is assumed as a lumped mass model. Under the mutual effects of the sway motions of the payload and the hoisting motion, the nonlinear behavior of the crane system is considered. A nonlinear control model-based scheme was designed to achieve the three objectives: (i) drive the crane system to the desired positions, (ii) suppresses the vibrations of the payload, and (iii) velocity tracking of hoisting motion. The nonlinear control scheme employs adaptation laws that estimate unknown system parameters, friction forces, and the mass of the payload. The estimated values were used to compute control forces applied to the trolley of the crane. The asymptotic stability of the crane system is investigated by using the Lyapunov method. The effectiveness of the proposed control scheme is verified by numerical simulation results.
\end{abstract}

Keywords: 3-D overhead crane; nonlinear adaptive control; Lyapunov method; Euler-Lagrange equation; sway control.

\section{INTRODUCTION}

Overhead crane systems are widely used to move goods from one place to another in factories and harbors. A crane is naturally an underactuated mechanical system, in which the number of actuators is less than degrees of freedom (DOF) of the system. For an overhead crane, the degree of freedom is five (i.e., trolley and girder positions, rope length, and two sway angles) but the number of actuators is three (i.e., trolley, girder, and hoisting-motors). To improve the efficiency of an overhead crane, the trolley and girder should travel as fast as possible. However, fast trolley and (or) girder motions resulted in the large sway motions of the payload. Therefore, the fast motions of the trolley and (or) girders do not guarantee the improvement of the overhead crane efficiency because it may take a long time to suppress the sway motions of the payload. Moreover, for safety, the sway motions should be

\footnotetext{
* Corresponding Author. Tel: +84-8-38688611
}

E-mail: nqchi@hcmut.edu.vn kept as small as possible during crane operation. Therefore, development of a control algorithm that allows fast trolley and girder motions together with quick sway suppression is desirable for crane systems.

A number of crane control algorithms based on 2D models [1-8] have been developed. It should be noted that $2 \mathrm{D}$ models do not represent all the cases of overhead crane operation in practice. 3D models [9-20] have been proposed to describe the crane more precisely, but they are more complex than 2D models. It should be noted that the complexity of the 3-D models yields difficulties in control design. Most researchers focused on 3D models of overhead cranes with four DOFs [914,17-18, 20] (i.e., trolley and girder, and two sway motions), where hoisting motion was not considered. It should be noted that the hoisting motion cannot be neglected in practice because the variation of the rope length significantly affects the sway dynamics. However, only few researches consider crane systems with five DOFs that include the hoisting motion [15-16] due to the 
complexity of the model and consequently control design.

Most of the researches about overhead crane in recent years are developed based on the EulerLagrange equation. Some of them use Lyapunov theory to design adaptive control [1-4,10-13, 16, 17] which estimates unknown parameters. The others use linearization technique in order to simplify the mathematical model and design of linear controllers such as sliding mode control [2, $7,8]$ and fuzzy control $[12,17]$.

In practice, the crane systems are usually operated under the unknown parameters such as the mass of payload and friction/damping force. To solve this problem, adaptive control [1-4,10-13, $16,17]$, fuzzy control $[12,17]$, and sliding mode control $[2,7,8]$ have been developed. Since adaptive control schemes are able to estimate unknown parameters that is used in control laws, Lyapunov energy-based control can be employed to establish the control design. The use of the Lyapunov control energy-based control facilitates the development of control algorithms based on nonlinear models, which represent nonlinear system precisely.

The work [7] proposed an adaptive sliding mode control using estimated unknown payload and damping coefficient, where the variation of the rope length is considered. However, the control adaptive scheme is based on a 2D model. In this paper, a 3D overhead crane model with five DOFs (motions of trolley and girder, hoisting, two sway angles) is derived using Euler-Lagrange equation. A nonlinear adaptive control that estimates the coefficients of friction and the mass of payload is proposed. The stability of the proposed control system is investigated using Lyapunov method. The effectiveness of the proposed control law is illustrated by experiment results.

\section{DYNAMICS OF A 3D OVERHEAD CRANE}

Figure 1 shows an overhead crane system with the sway motions of the payload in the world coordinate system OXYZ. In the derivation of the dynamic model of the crane, the following assumptions are made:

(i) The payload and the trolley are connected by a massless-rigid link. Also, the mechanical frame of the crane is considered as a rigid body.

(ii) The mass of trolley is unknown.

(iii) The friction forces $f_{c x}$ and $f_{c y}$ cannot be measured, where the viscous friction coefficients $c_{x}$ and $c_{y}$ are unknown.



Figure 1. The overhead crane system

As shown in Figure 1, the girder, the trolley, and the payload position vectors are given as follows

$r_{r}=x \quad 0 \quad 0$,

$r_{c}=x \quad y \quad 0$,

$r_{p}=x+l \sin \theta \cos \phi \quad y+l \sin \phi \quad-l \cos \theta \cos \phi$,

where $x$ and $y$ are the trolley position in $X$ and $Y$ directions, respectively.

Let $q(t) \in R^{5}$ be the generalized coordinate vector defined as follows.

$$
q(t)=x(t) \quad y(t) \quad l(t) \quad \theta(t) \quad \phi(t)^{\mathrm{T}}
$$

The forces applied to the system are given by.

$$
F=\left[\begin{array}{lllll}
\left(F_{x}-f_{c x}\right) & \left(F_{y}-f_{c y}\right) & F_{l} & 0 & 0
\end{array}\right](3)
$$

The friction forces in the $\mathrm{X}$ and $\mathrm{Y}$ directions respective are given as follows.

$$
f_{c x}(t)=c_{x} \dot{x}(t), f_{c y}(t)=c_{y} \dot{y}(t)
$$

where $c_{x}$ and $c_{y}$ are the viscous friction coefficients in $X$ and $Y$ directions, respectively.

The total kinetic energy $K$ and the potential energy $P$ of the crane system are given as

$$
\begin{aligned}
& K=K_{\text {trolley }}+K_{\text {rail }}+K_{\text {payload }}, \\
& P=P_{\text {payload }},
\end{aligned}
$$

where

$$
\begin{aligned}
& K_{\text {trolley }}=\frac{1}{2} m_{c} \dot{r}_{c} \cdot \dot{r}_{c} \\
& K_{\text {rail }}=\frac{1}{2} m_{r} \dot{r}_{r} \cdot \dot{r}_{r}, \\
& K_{\text {payload }}=\frac{1}{2} m_{p} \dot{r}_{p} \cdot \dot{r}_{p}, \\
& P_{\text {payload }}=m_{p} g l(1-\cos \theta \cos \phi) .
\end{aligned}
$$


Using the Euler-Lagrange equation [21], the equations of motion are derived as follows

$$
\frac{d}{d t}\left(\frac{\partial L}{\partial \dot{q}_{i}}\right)-\frac{\partial L}{\partial q_{i}}=T_{i} \quad(i=1,2,3,4,5)
$$

with $L=K-P$.

The dynamic equations (10) can be rewritten as

$$
M(q) \ddot{q}+C_{m}(q, \dot{q}) \dot{q}+G(q)=u,
$$

where $M(q) \in R^{5 \times 5}$ is inertia matrix of the crane system and $C_{m}(q) \in R^{5 \times 5}$ represent the centripetal Coriolis, and $G(q) \in R^{5}$ is the gravity term. Based on the structure of $M(q)$ and $C_{m}(q, \dot{q})$ given by Eq. (11), it should be noted that the following skew-symmetric relationship is satisfied.

$$
\xi^{T}\left(\frac{1}{2} \dot{M}(q)-C_{m}(q, \dot{q})\right) \xi=0, \quad \forall \xi \in R^{5},
$$

where $M(q)$ can be upper and lower bounded by the following inequality.

$$
n_{1}\|\xi\|^{2} \leq \xi^{T} M(q) \xi \leq n_{2}\|\xi\|^{2}, \forall \xi \in R^{5}
$$

where $n_{1}$ and $n_{2} \in R$ are positive bounded constants.

\section{Control DeSign}

In this section, a control law is proposed to drive the crane to the desired position and to suppress the sway angles simultaneously. For convenience, a new generalized coordinate vector is defined as follows

$$
q^{\mathrm{T}}=q_{m} \quad q_{a},
$$

where

$$
q_{m}=x(t) y(t) l(t)^{T}, q_{a}=\theta(t) \phi(t)^{T} .
$$

The equations of motion of the overhead crane (11) can be rewritten as follow.

$$
\begin{aligned}
& {\left[\begin{array}{cc}
M_{m m} & M_{m a} \\
M_{a m} & M_{a a}
\end{array}\right]\left[\begin{array}{l}
\ddot{q}_{m} \\
\ddot{q}_{a}
\end{array}\right]+\left[\begin{array}{cc}
C_{m m} & C_{m a} \\
C_{a m} & C_{a a}
\end{array}\right]\left[\begin{array}{c}
\dot{q}_{m} \\
\dot{q}_{a}
\end{array}\right]+\left[\begin{array}{c}
G_{m}(q) \\
G_{a}(q)
\end{array}\right]} \\
& =\left[\begin{array}{c}
u_{m f} \\
0
\end{array}\right]-\left[\begin{array}{c}
u_{m c f} \\
0
\end{array}\right] .
\end{aligned}
$$

To achieve the control objective, with the given desired signals $q_{d}, \dot{q}_{d}$, and $\ddot{q}_{d}$ (which are assumed to be bounded), the control law $u_{m f}$ is designed to guarantee the asymptotical convergence of $q$ to $\dot{q}_{d}$.
The error signals are defined as

$$
e=q-q_{d}=\left[\begin{array}{ll}
e_{m}^{T} & e_{a}^{T}
\end{array}\right]^{T},
$$

where

$$
\begin{aligned}
& e_{m}=q_{m}-q_{d m}=\left[\begin{array}{lll}
e_{x} & e_{y} & e_{l}
\end{array}\right]^{\mathrm{T}} \\
& e_{a}=q_{a}-q_{d a}=\theta-\theta_{d} \quad \phi-\phi_{d}{ }^{\mathrm{T}}=\left[\begin{array}{ll}
e_{\theta} & e_{\phi}
\end{array}\right]^{\mathrm{T}}
\end{aligned}
$$

where $x_{d}, y_{d}, l_{d}, \theta_{d}, \phi_{d}$ are defined trajectories of $x, y, L, \theta, \phi$, respectively. $\dot{q}_{r}$ are defined as follows

$$
\dot{q}_{r}=\dot{q}_{d}-K e=\left[\begin{array}{c}
\dot{q}_{d m}-K_{m} e_{m} \\
\dot{q}_{d a}-K_{a} e_{a}
\end{array}\right]=\left[\begin{array}{c}
\dot{q}_{r m} \\
\dot{q}_{r a}
\end{array}\right]=\left[\begin{array}{c}
\dot{q}_{r x} \\
\dot{q}_{r y} \\
\dot{q}_{r l} \\
\dot{q}_{r \theta} \\
\dot{q}_{r \phi}
\end{array}\right],
$$

where

$$
K=\left[\begin{array}{ccc}
K_{m} & 0 & 0 \\
0 & 0 & 0 \\
0 & 0 & K_{a}
\end{array}\right], K=\left[\begin{array}{ccc}
K_{1} & 0 & 0 \\
0 & K_{2} & 0 \\
0 & 0 & K_{3}
\end{array}\right], K=\left[\begin{array}{cc}
K_{4} & 0 \\
0 & K_{5}
\end{array}\right]
$$

$K_{m}$ and $K_{a}$ are positive definite matrices. A new variable $s$ are defined as follows

$$
s=\dot{q}-\dot{q}_{r}=\left[\begin{array}{c}
\dot{q}_{m}-\dot{q}_{r m} \\
\dot{q}_{a}-\dot{q}_{r a}
\end{array}\right]=\left[\begin{array}{c}
s_{m} \\
s_{a}
\end{array}\right]
$$

Then, using Eq. (11), the dynamics in terms of the signals $s_{m}$ and $s_{a}$ can be derived as

$$
\left[\begin{array}{cc}
M_{m m} & M_{m a} \\
M_{a m} & M_{a a}
\end{array}\right]\left[\begin{array}{c}
\dot{s}_{m} \\
\dot{s}_{a}
\end{array}\right]+\left[\begin{array}{cc}
C_{m m} & C_{m a} \\
C_{a m} & C_{a a}
\end{array}\right]\left[\begin{array}{c}
s_{m} \\
s_{a}
\end{array}\right]=\left[\begin{array}{c}
\tau_{m}+u_{m f} \\
\tau_{a}
\end{array}\right],
$$

where the fictitious torques $\tau_{m}$ and $\tau_{a}$ are defined as follows

$$
\begin{aligned}
\tau_{m}= & M_{m m}-\ddot{q}_{r m}+M_{m a}-\ddot{q}_{r a}+C_{m m}-\dot{q}_{r m} \\
& +C_{m a}-\dot{q}_{r a}-G_{m}(q)-u_{m c f}, \\
\tau_{a}= & M_{a m}-\ddot{q}_{r m}+M_{a a}-\ddot{q}_{r a}+C_{a m}-\dot{q}_{r m} \\
& +C_{a a}-\dot{q}_{r a}-G_{a}(q) .
\end{aligned}
$$

The signals $\tau_{m}$ and $\tau_{a}$ can be expressed as in term of a known matrix $\omega_{m}$ and $\omega_{a}$ and unknown parameter vectors, $\psi_{m}$ and $\psi_{a}$.

$$
\begin{aligned}
\boldsymbol{\tau}_{m} & =\left[\begin{array}{ccccc}
\tau_{m 11} & 0 & \tau_{m 13} & \dot{x} & 0 \\
0 & \tau_{m 22} & \tau_{m 23} & 0 & \dot{y} \\
0 & 0 & \tau_{m 32} & 0 & 0
\end{array}\right]\left[\begin{array}{c}
m_{c}+m_{r}+m_{p} \\
m_{c}+m_{p} \\
m_{p} \\
c_{x} \\
c_{y}
\end{array}\right] \\
& =\omega_{m} \psi_{m},
\end{aligned}
$$


where

$$
\begin{aligned}
\tau_{m 11}= & -\ddot{q}_{r x} \\
\tau_{m 13}= & -\sin \theta \cos \phi \ddot{q}_{r l}-l \cos \theta \cos \phi \ddot{q}_{r \theta} \\
& +l \sin \theta \sin \phi \ddot{q}_{r \phi}-(\cos \theta \cos \phi \dot{\theta}-\sin \theta \sin \phi \dot{\phi}) \dot{q}_{r l} \\
& -\cos \theta \cos \phi \dot{l}-l \sin \theta \cos \phi \dot{\theta}-l \cos \theta \sin \phi \dot{\phi} \dot{q}_{r \theta} \\
& +\sin \theta \sin \phi \dot{l}+l \cos \theta \sin \phi \dot{\theta}+l \sin \theta \cos \phi \dot{\phi} \dot{q}_{r \phi} \\
\tau_{m 22}= & -\ddot{q}_{r y} \\
\tau_{m 23}= & -\sin \phi \ddot{q}_{r l}-l \cos \phi \ddot{q}_{r \phi}-\cos \phi \dot{\phi} \dot{q}_{r l} \\
& -\cos \phi \dot{l}-l \sin \phi \dot{\phi} \dot{q}_{r \phi} \\
\tau_{m 32}= & -\sin \theta \cos \phi \ddot{q}_{r x}-\sin \phi \ddot{q}_{r y}-\ddot{q}_{r l} \\
& +l \cos { }^{2} \phi \dot{\theta} \dot{q}_{r \theta}+l \dot{\phi} \dot{q}_{r \phi}+g-g \cos \theta \cos \phi . \\
\tau_{a} & =\left[\begin{array}{ll}
\tau_{a 11} & 0 \\
0 & \tau_{a 22}
\end{array}\right]\left[\begin{array}{l}
m_{p} \\
m_{p}
\end{array}\right]=\omega_{a} \psi_{a},
\end{aligned}
$$

where

$$
\begin{aligned}
\tau_{a 11}= & -l \cos \theta \cos \phi \ddot{q}_{r x}-l^{2} \cos ^{2} \phi \ddot{q}_{r y}-l \cos ^{2} \phi \dot{\theta} \dot{q}_{r l} \\
& -l \cos ^{2} \phi \dot{l}-l^{2} \sin \phi \cos \phi \dot{\phi} \dot{q}_{r \theta} \\
& +l^{2} \cos \phi \sin \phi \dot{\theta} \dot{q}_{r \phi}-g l \sin \theta \cos \phi \\
\tau_{a 11}= & -l \cos \theta \cos \phi \ddot{q}_{r x}-l^{2} \cos ^{2} \phi \ddot{q}_{r y}-l \cos ^{2} \phi \dot{\theta} \dot{q}_{r l} \\
& -l \cos { }^{2} \phi \dot{l}-l^{2} \sin \phi \cos \phi \dot{\phi} \dot{q}_{r \theta} \\
& +l^{2} \cos \phi \sin \phi \dot{\theta} \dot{q}_{r \phi}-g l \sin \theta \cos \phi
\end{aligned}
$$

As a majority of the adaptive controller, the following signal is defined.

$$
\dot{Z}_{x}=\left\{\begin{array}{lr}
2\left(\sqrt{Z_{x}} a_{x}(t)+b_{x}(t)\right), \quad Z_{x}(t)>0 \\
2 b_{x}(t) & Z_{x}(t)=0 \wedge b_{x}(t)>0 \\
\delta_{x}, & Z_{x}(t)=0 \wedge b_{x}(t) \leq 0
\end{array}\right.
$$

where $\delta_{x}$ is a positive constant and

$$
\begin{aligned}
& a_{x}(t)=\frac{\left\|s_{m}\right\|^{2}}{\left\|s_{m}\right\|^{2}+\varepsilon}-s_{a}{ }^{T} \omega_{a} \hat{\psi}_{a}-s_{a}{ }^{T} K_{a v} s_{a} \\
& b_{x}(t)=\frac{\varepsilon}{\left\|s_{m}\right\|^{2}+\varepsilon}-s_{a}{ }^{T} \omega_{a} \hat{\psi}_{a}-s_{a}{ }^{T} K_{a v} s_{a}
\end{aligned}
$$

From Eq. (24), it is concluded that $Z_{x}(t)$ is positive. Define a positive function $h(t)=\sqrt{Z_{x}}$. It can be shown that

$$
\begin{aligned}
& \dot{h}(t)=\frac{1}{h(t)}\left(\frac{h\left\|s_{m}\right\|^{2}+\varepsilon}{\left\|s_{m}\right\|^{2}+\varepsilon}-s_{a}{ }^{T} \omega_{a} \hat{\psi}_{a}-s_{a}^{T} K_{a v} S_{a}\right) \\
& \forall h(t) \neq 0
\end{aligned}
$$

It is assumed that there exists a measure zero set of time sequences $t_{i}{ }_{i=1}^{\infty}$ such that $Z\left(t_{i}\right)=0$ (i.e., $h\left(t_{i}\right)=0, i=1,2,3, \ldots, \infty$ ).

The following control law is proposed.

$$
u_{m f}=-\omega_{m} \hat{\psi}_{m}-\tau_{v}-K_{m v} s_{m}
$$

where

$$
\tau_{v}=\left[\frac{(h-1) s_{m}}{\left\|s_{m}\right\|^{2}+\varepsilon}\right]-s_{a}{ }^{T} \omega_{a} \hat{\psi}_{a}-s_{a}{ }^{T} K_{a v} s_{a}
$$

where $\hat{\psi}_{m}$ and $\hat{\psi}_{a}$ are the estimates of $\psi_{m}$ and $\psi_{a}$ , respectively.

The adaptation laws are given as.

$$
\left\{\begin{array}{l}
\dot{\hat{\psi}}_{m}=s_{m}^{T} \omega_{m} \lambda_{m} \\
\dot{\hat{\psi}}_{a}=s_{a}^{T} \omega_{a} \lambda_{a}
\end{array}\right.
$$

Then the error dynamics can be obtained as.

$$
\begin{aligned}
& {\left[\begin{array}{cc}
M_{m m} & M_{m a} \\
M_{a m} & M_{a a}
\end{array}\right]\left[\begin{array}{l}
\dot{s}_{m} \\
\dot{s}_{a}
\end{array}\right]+\left[\begin{array}{ll}
C_{m m} & C_{m a} \\
C_{a m} & C_{a a}
\end{array}\right]\left[\begin{array}{c}
s_{m} \\
s_{a}
\end{array}\right]+\left[\begin{array}{cc}
K_{m v} & 0 \\
0 & K_{a v}
\end{array}\right]\left[\begin{array}{l}
s_{m} \\
s_{a}
\end{array}\right]} \\
& =\left[\begin{array}{l}
-\omega_{m} \tilde{\psi}_{m}-\tau_{v} \\
\omega_{a} \psi_{a}+K_{a v} s_{a}
\end{array}\right],
\end{aligned}
$$

which can be rewritten as follows.

$$
M(q) \dot{s}+C_{m}(q, \dot{q}) s+K s=\left[\begin{array}{c}
-\omega_{m} \tilde{\psi}_{m}-\tau_{v} \\
\omega_{a} \psi_{a}+K_{a v} s_{a}
\end{array}\right]
$$

where

$$
\left[\begin{array}{c}
\tilde{\psi}_{m} \\
\tilde{\psi}_{a}
\end{array}\right]=\left[\begin{array}{c}
\hat{\psi}_{m}-\psi_{m} \\
\hat{\psi}_{a}-\psi_{a}
\end{array}\right]
$$

Since $\hat{\psi}_{m}$ and $\hat{\psi}_{a}$ are constant, we obtain.

$$
\left[\begin{array}{c}
\dot{\tilde{\psi}}_{m} \\
\dot{\tilde{\psi}}_{a}
\end{array}\right]=\left[\begin{array}{c}
\dot{\hat{\psi}}_{m}-\dot{\psi}_{m} \\
\dot{\hat{\psi}}_{a}-\dot{\psi}_{a}
\end{array}\right]=\left[\begin{array}{c}
\dot{\hat{\psi}}_{m} \\
\dot{\hat{\psi}}_{a}
\end{array}\right] .
$$

Theorem: Consider the system (11) under the parameters systems unknown. The proposed control law (26) employing the adaption laws (27) guarantees the asymptotic stability of the systems, i.e., $s \rightarrow 0, e \rightarrow 0$, and $\dot{e} \rightarrow 0$ as $t \rightarrow \infty$.

Proof: Lyapunov function candidate can be defined as.

$$
V(t)=\frac{1}{2} s^{T} M(q) s+\frac{1}{2} \tilde{\psi}_{m}{ }^{T} \lambda_{m}^{-1} \tilde{\psi}_{m}+\frac{1}{2} \tilde{\psi}_{a}{ }^{T} \lambda_{a}^{-1} \tilde{\psi}_{a}+\frac{1}{2} Z_{x}
$$


From Eqs. (23)-(24), $V(t)$ positive-definite can be concluded. Taking the time derivative of $V(t)$ yields.

$$
\begin{aligned}
\dot{V}(t)= & -s^{T} K s+ \\
& -s_{m}{ }^{T} \omega_{m}+\dot{\tilde{\psi}}_{m}{ }^{T} \lambda_{m}^{-1} \tilde{\psi}_{m}+\dot{\tilde{\psi}}_{a}{ }^{T} \lambda_{a}^{-1}-s_{a}{ }^{T} \omega_{a} \tilde{\psi}_{a} .
\end{aligned}
$$

By substituting (27) into (32), the following inequality is obtained.

$$
\dot{V}(t)=-s^{\mathrm{T}} K s \leq 0
$$

From Eq. (23), it is concluded that $h(t)$ is continuous for all $t_{i}$. Since $V(t)$ is a continuous function of $h(t), V(t)$ is continuous at time $t_{i}$, i.e., $V\left(t_{i}^{-}\right)=V\left(t_{i}^{+}\right)$. From Eq. (33), $\dot{V}\left(t_{i}^{-}\right)<0$ and $\dot{V}\left(t_{i}^{+}\right)<0$ then it is concluded that $\dot{V}(t)$ is nonincreasing at time $t_{i}$, which implies $s, h \in L_{\infty}$. Therefore, from Eq. (22) $e, \tau_{m}, \tau_{a} \in L_{\infty}$, and using definitions of $\tau_{m}, \tau_{a}, \dot{s} \in L_{\infty}$ is obtained. Moreover, it is clear that $\int_{0}^{\infty} \dot{V} d t=V(\infty)-\mathrm{V}(0)<\infty$, or equivalent. Therefore by invoking the Barbalat's lemma, we obtain that $\mathbf{s} \rightarrow 0$ asymptotically as $t \rightarrow \infty$, therefore, implies $\mathbf{e}$ and $\dot{\mathbf{e}} \rightarrow 0$ as $t \rightarrow \infty$.

\section{EXPERIMENT RESULTS}

The experiments were carried out with the testbed, as shown in Figure 2. The payload hangs at the end of the rope whose top end is hinged by the pulley mounted on the trolley. Three AC servo motors (MITSUBISHI MR-J2S-40) make the three motions of the crane system: the trolley, the girder, and the hoisting. The three motors are controlled via current inputs provided by a power interface.

The five encoders are employed to measure the five variables, i.e. the displacements of the trolley and the gantry, the rope length, and the two sway angles of the payload, where three encoders are available in the three AC servo motors. The pulse signals of the five encoders are converted to 16-bit digital signals by the power interface.

The reference signals for the three AC servo motors are sent from a $\mathrm{PC}$ to the power interface through a PCI board (SMC-4DF-PCI provided by CONTEC company), which also receives the 16bit digital signals of the five encoders. The control program runs in Windows 7 environment.

To illustrate the control performance, we perform the experiments of the proposed nonlinear adaptive controller of (30) employing the adaptation laws (27) in a testbed (as shown in Figure 2) with the following parameters:

$$
\begin{aligned}
& m_{c}=5 \mathrm{~kg}, m_{r}=5 \mathrm{~kg}, \\
& m_{p}=0.5 \mathrm{~kg}, g=9.81 \mathrm{~m} / \mathrm{s}^{2}
\end{aligned}
$$

The initial state of the system is chosen as:

$$
\begin{aligned}
& x(0)=0, \dot{x}(0)=0, \mathrm{y}(0)=0, \dot{y}(0)=0, \\
& l(0)=1 \mathrm{~m}, \dot{i}(0)=0, \theta(0)=0, \dot{\theta}(0)=0, \\
& \phi(0)=0, \dot{\phi}(0)=0 .
\end{aligned}
$$

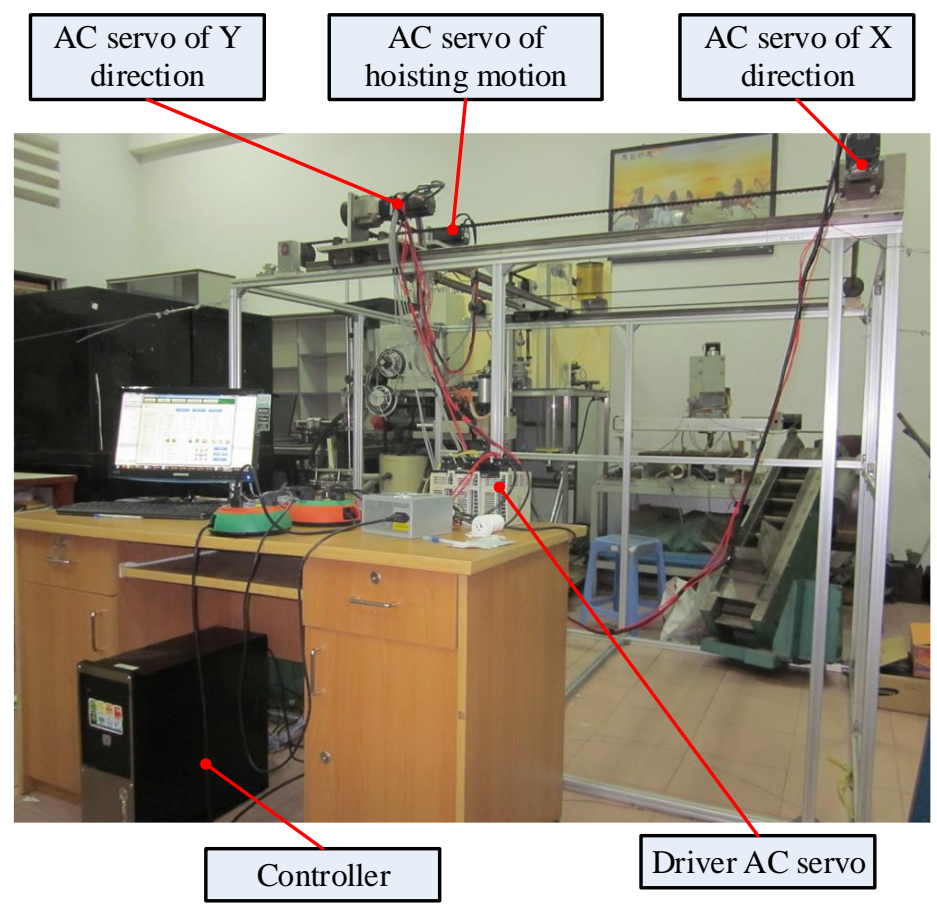

Figure 2. Experimental system 
The trolley moves to the desired position selected as follows:

$$
\begin{aligned}
& x_{d}=0.8 \mathrm{~m}, \quad \dot{x}_{d}=0, \\
& y_{d}=0.8 \mathrm{~m}, \quad \dot{y}_{d}=0, \\
& l_{d}=0 \mathrm{~m}, \quad \dot{l}_{d}=0 .
\end{aligned}
$$

The control gains of the control law (26) are turned until the best performance is achieved, which yields the following control gains.

$$
\begin{aligned}
K_{m} & =\left[\begin{array}{ccc}
17 & 0 & 0 \\
0 & 15 & 0 \\
0 & 0 & 15
\end{array}\right], K_{a}=\left[\begin{array}{cc}
0.1 & 0 \\
0 & 0.1
\end{array}\right], \\
K_{v m} & =\left[\begin{array}{ccc}
1.2 & 0 & 0 \\
0 & 1.5 & 0 \\
0 & 0 & 3.5
\end{array}\right], K_{v a}=\left[\begin{array}{cc}
0.6 & 0 \\
0 & 0.6
\end{array}\right],
\end{aligned}
$$

$\lambda_{m}=0.02, \quad \lambda_{a}=0.2, \quad \varepsilon=0.03$

Figures 3, 4 and 5 plots demonstrate that trolley reach $x_{d}=0.8 \mathrm{~m}$ at $t=12.3 \mathrm{~s}$ and $y_{d}=0.8 \mathrm{~m}$ at $t=$ $12.3 \mathrm{~s}$, the rope will drop payload down from initial height $l(0)=0.8 \mathrm{~m}$ to zero position after duration $t$ $=12.3 \mathrm{~s}$. During trolley is moving, the maximum

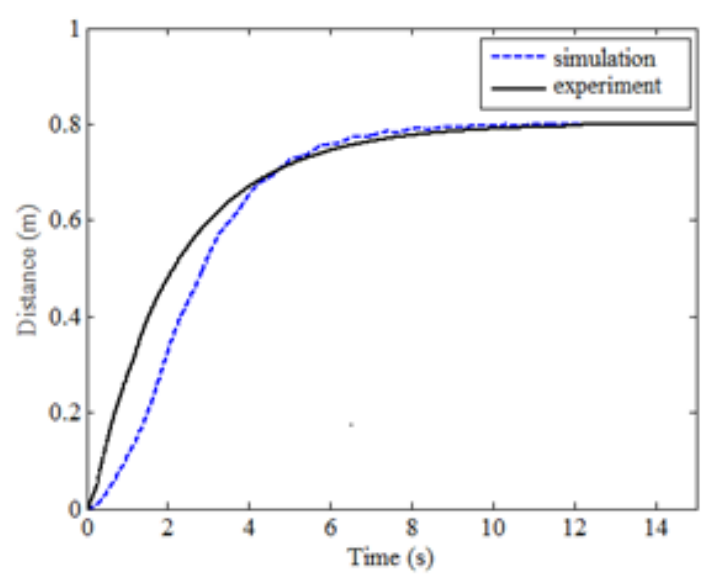

Figure 3. Position of the trolley in $X$-direction

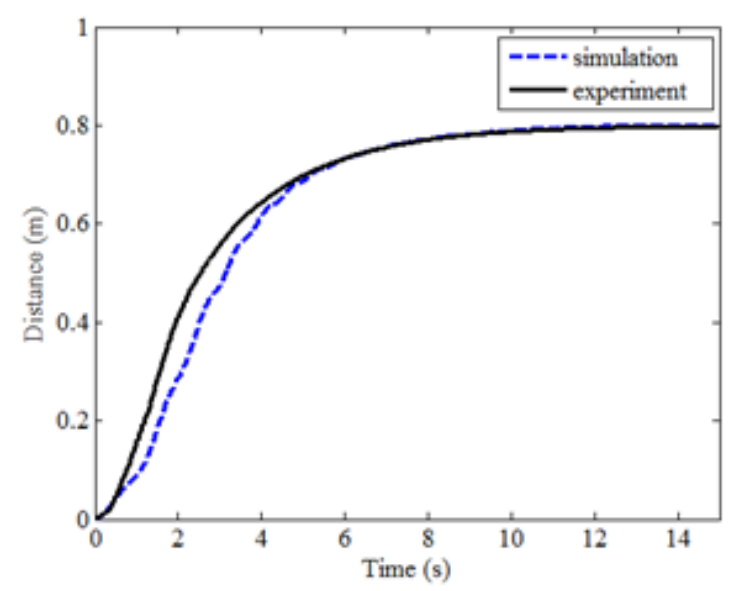

Figure 4. Position of the trolley in $Y$-direction

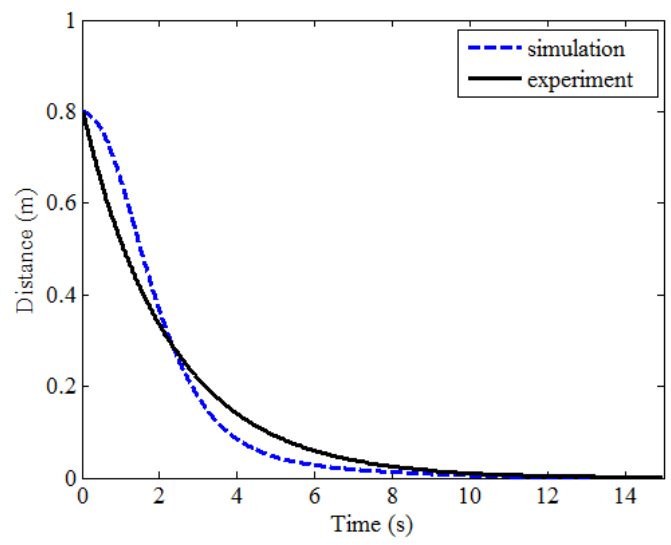

Figure 5. Rope length

vibration amplitudes of the sway angles $\left|\theta_{\max }(t)\right|=4^{0}$ and $\left|\phi_{\max }(t)\right|=3.3^{0}$ are demonstrated in Figure 6 and 7. After 14 seconds, the vibration almost eliminated

Figures 8 and 9 are the parameters estimation results. The estimated values converge to constant although it may not get the true values. As shown in Figures 8 and 9, the values may not get the true values. However, getting true values of the parameters was not the purpose of this paper.

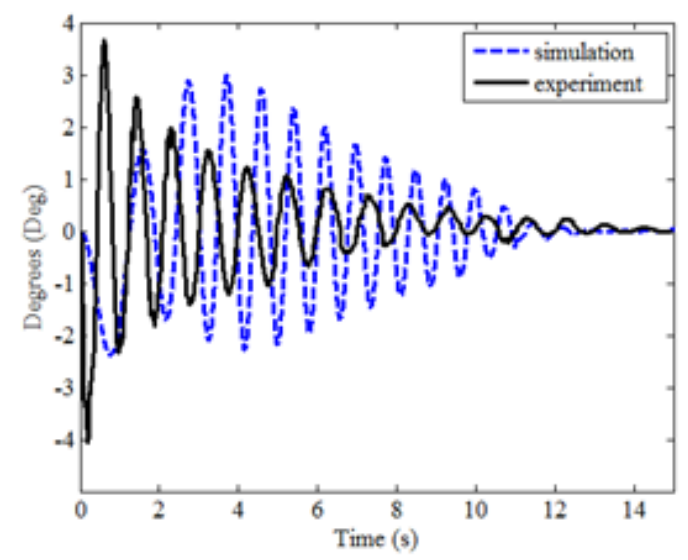

Figure 6. Sway angle $\theta(t)$



Figure 7. Sway angle $\phi(t)$ 


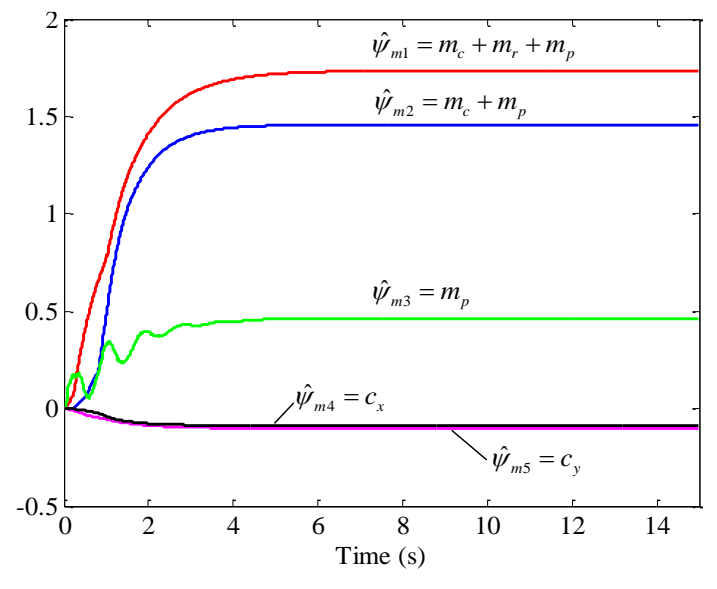

Figure 8. Estimated parameters $\hat{\psi}_{m}(t)$

\section{Conclusion}

In this paper, a 5-DOF dynamic model of the 3D overhead crane was developed under the effects of unknown friction force and unknown payload. A nonlinear adaptive controller was proposed for the overhead crane to drive it to its desired point and to suppress the swing of payload. Under the proposed controller, asymptotic stability of the overhead crane system is proved by using Lyapunov method. Experiment and simulation results illustrate the effectiveness of the proposed controller.

\section{ACKNOWLEDGEMENT}

This research is funded by Vietnam National Foundation for Science and Technology Development (NAFOSTED) under grant number 107.04-2012.37 and by Vietnam National University Ho Chi Minh City (VNU-HCM) under grant number C2013-20-01.

\section{REFERENCES}

[1] D. Liu et al., "Adaptive sliding mode fuzzy control for a 2D overhead crane," Mechatronics, vol. 15, no. 5, pp. 505-522, 2005.

[2] H. H. Lee et al., "A sliding mode anti-swing trajectory control for overhead cranes with high speed load hoisting," Journal of Dynamic Systems, Measurement and Control, vol. 128, no. 4, pp. 842-845, 2006.

[3] C. Y. Chang, "Adaptive fuzzy controller of the overhead cranes with nonlinear disturbance," IEEE Transactions on Industrial Informatics, vol. 3, no. 2, pp. 164 172, 2007.

[4] M. S. Park et al., "Anti-sway tracking control of overhead cranes with system uncertainty and actuator nonlinearity using an adaptive

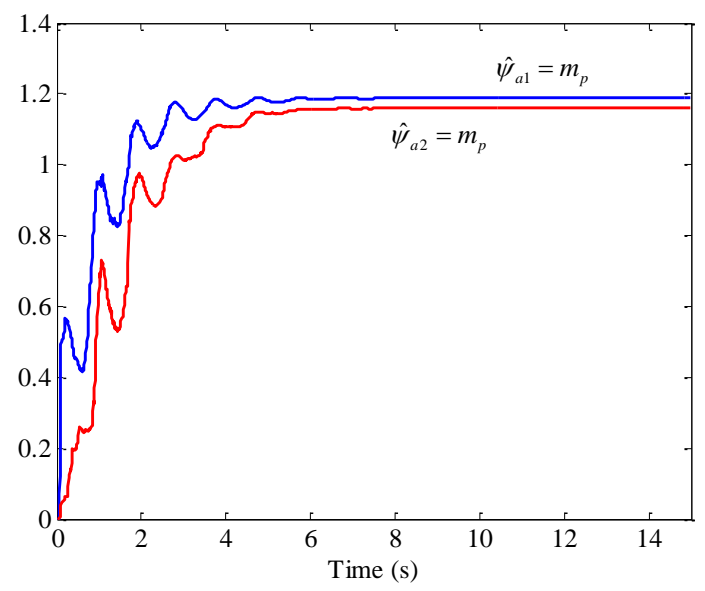

Figure 9. Estimated parameters $\hat{\psi}_{a}(t)$

fuzzy sliding mode control," IEEE Transactions on Industrial Electronics, vol. 55, no. 11, pp. 3972-3984, 2008.

[5] S. W. Su et al., J. Zhu, D. B. Lowe, P. B. McLean, S. Huang, N. Nguyen, R. S. Nicholson and K. Wing, "Model predictive control of gantry crane with input nonlinearity compensation," in World Academy of Science, Engineering and Technology, 2009.

[6] Y. Fang et al., "A motion planning based adaptive control method for an under actuated crane system," IEEE Transactions on Control Systems Technology, vol. 20, no. 1, pp. 241-248, 2011.

[7] L. A. Tuan et al., "Adaptive sliding mode control of overhead cranes with varying cable length," Journal of Mechanical Science and Technology, vol. 27, no. 3, pp. 885-893, 2013.

[8] D. Qian and J. Yi, "Design of combining sliding mode controller for overhead crane systems," International Journal of Control and Automation, vol. 6, no. 1, 2013.

[9] H. Chen et al., "Dynamical modeling and nonlinear control of a 3D crane," in Proc. of the International Conference on Control and Automation, 2005, pp. 1085-1090.

[10] J. H. Yang and K. S. Yang, "Adaptive control for 3-D overhead crane systems," in Proc. Of the American Control Conference, 2006.

[11] K. S. Lee and H. C. Cho, "Adaptive control and stability analysis of nonlinear crane systems with perturbation," Journal of Mechanical Science and Technology, vol. 22, no. 6, pp. 1091-1098, 2008.

[12] C. Y. Chang and K. H. Chiang, "Fuzzy projection control law and its application to the overhead crane," Mechatronics, vol. 18, no. 10, pp. 607-615, 2008. 
[13] J.-H. Yang, Linear Adaptive control-Chapter 13: on the adaptive tracking control of 3-D overhead crane system, Intech, DOI: 10.5772/6511, 2009.

[14] N. B. Almutairi and M. Zribi, "Sliding mode control of a three-dimensional overhead crane," Journal of Vibration and Control, vol. 15, no. 11, pp. 1679-1730, 2009.

[15] A. Pisano et al., "Load swing suppression in the 3-dimensional overhead crane via second-order sliding-modes," in Proc. Of the $201011^{\text {th }}$ International Workshop on Variable Structure Systems (VSS), pp. 452457, 2010.

[16] J. H. Yang and S. H. Shen, "Novel approach for adaptive tracking control of a 3-D overhead crane system," Journal of Intelligent \& Robotic Systems, vol. 62, no. 1, pp. 59-80, 2010.

[17] W. Yu, M. A. M. Armendariz and F. O. Rodriguez, "Stable adaptive compensation with fuzzy CMAC for an overhead crane,"
Information Sciences, vol. 181, no. 21, pp. 4895-4907, 2011.

[18] L. A. Tuan et al., "Partial feedback linearization control of a three-dimensional overhead crane," International Journal of Control, Automation and Systems, vol. 11, no. 4, pp. 718-727, 2013.

[19] R. M. T. R. Ismail et al., "Nonlinear dynamic modeling and analysis of a 3-D overhead gantry crane system with payload variation," in Proc. Of UKSim Third European Modeling Symposium on Computer Modeling and Simulation, pp. 350-354, 2009.

[20] Q. H. Ngo and K. S. Hong, "Sliding-mode anti-sway control of an offshore container crane," IEEE/ASME Transactions on Mechatronics, vol. 17, no. 2, pp. 662-668, 2012.

[21] S-J. Ying, "Dynamics of mechanical systems," in Plastics, Ed.Virginia: American Insitute of Areonautics Astronautics, 1997, pp. 55-56 